\title{
Metodologías para el cálculo de emisiones de gases de efecto invernadero: descripción del proyecto de control en una industria productora de cemento
} Methodologies for the calculation of greenhouse gas emissions: description of the control project in a cement-producing Industry

María Concepción Martínez-Rodríguez ${ }^{1}$, Lorena Elizabeth Campos-Villegas ${ }^{2}$, Jesús Castillo-Monroy ${ }^{3}$

Martínez-Rodríguez, M.C; Campos-Villegas, L.E; CastilloMonroy, J. Metodologías para el cálculo de emisiones de gases de efecto invernadero: descripción del proyecto de control en una industria productora de cemento. Tecnología en Marcha. Vol. 34-3 Julio-Setiembre 2021. Pág 177-189.

doi) https://doi.org/10.18845/tm.v34i3.5313

1 Instituto Politécnico Nacional, Centro Interdisciplinario de Investigaciones y Estudios sobre Medio Ambiente y Desarrollo. México. Correos electrónicos: mcmartinezr@ipn.mx; mconcepcionmr@yahoo.com.mx (iD https://orcid.org/0000-0003-3094-5411

2 Instituto Politécnico Nacional, Centro Interdisciplinario de Investigaciones y Estudios sobre Medio Ambiente y Desarrollo. México. Correo electrónico: lor ca2003@yahoo.com.mx y Estudios sobre Medio Ambiente y Desarrollo. México. Correo electrónico: josjes.castillo@hotmail.com 


\title{
Palabras clave
}

Políticas públicas ambientales; cambio climático; industria cementera; gases de efecto invernadero; auditoria.

\section{Resumen}

La industria cementera global, es responsable de aproximadamente el $5 \%$ del total de emisiones de $\mathrm{CO}_{2}$ en el mundo, como resultado de las reacciones químicas en la producción de clinker, el consumo de combustible, el uso de electricidad, el transporte de materias primas y productos terminados. El objetivo general de la presente investigación es proporcionar las bases metodológicas de preparación del proyecto de control de emisiones con vista a una auditoria inicial para Gases de Efecto Invernadero (GEI) en una Industria del sector cemento con la finalidad de identificar el nivel de gestión de sus sistemas y procesos para el cálculo de los GEl alcance 1 y 2 así como sugerir un procedimiento de cálculo de GEl que fortalezca los procesos de reporte de las emisiones GEI. Este trabajo se basó en la ISO 14064-2, relativa a la Especificación, a nivel de proyecto, para la cuantificación, el seguimiento y la base para definir el informe de la reducción de emisiones o el aumento en las remociones de gases de efecto invernadero. Las principales variables a considerar en la validación del inventario de emisiones de GEl que se sugiere considerar en el marco de la auditoria teniendo en primer nivel; de fijar al interior de la empresa el año inicial o cero, e integrar un compendio histórico de al menos 5 años de las emisiones de GEl, estimar las emisiones por calcinación de materia prima, por uso de combustibles y producción de cemento.

\section{Keywords}

Environmental public policies; climate change; cement industry; greenhouse gases; audit.

\begin{abstract}
The global cement industry is responsible for approximately $5 \%$ of total $\mathrm{CO}_{2}$ emissions in the world, as a result of chemical reactions in clinker production, fuel consumption, electricity use, transportation of raw materials and finished products. The general objective of this research is to provide the methodological bases for the preparation of the emission control project with a view to an initial audit for Greenhouse Gases (GHG) in a Cement industry in order to identify the management level of its systems and processes for calculating GHG scope 1 and 2 and suggest a GHG calculation procedure that strengthens the GHG emissions reporting processes. This work was based on the ISO 14064-2, regarding the Specification, at the project level, for the quantification, monitoring and the basis for defining the report of the reduction of emissions or the increase in greenhouse gas removals. The main variables to consider in the validation of the inventory of GHG emissions that are suggested will be considered in the framework of the audit taking first level; to set within the company the initial year or zero, and to integrate a historical compendium of at least 5 years of GHG emissions, to estimate emissions from calcination of raw material, by use of fuels and cement production.
\end{abstract}

\section{Introducción}

El cambio climático representa hoy día uno de los mayores desafíos a los que se enfrentan los países, los gobiernos, las empresas y la humanidad del siglo XXI, y uno de los principales factores que incide sobre este fenómeno es la generación de Gases de Efecto Invernadero (GEI) debido a las actividades inherentes a los procesos de producción. La acumulación de gases 
de efecto invernadero (GEI) en la atmósfera está provocando un aumento de la temperatura global (calentamiento global), que es extremadamente amenazante para la vida en la tierra, desde la revolución industrial, como consecuencia de la expansión industrial y la quema de combustibles fósiles, el nivel de GEl en la atmósfera aumentó sustancialmente y se espera que aumente aún más en el futuro. En comparación con 1750, las concentraciones de dióxido de carbono $\left(\mathrm{CO}_{2}\right)$ en la atmósfera han aumentado de 280 a 410 ppmV, y esta tendencia se espera en las próximas décadas, resultando un aumento de temperatura de hasta $5.8^{\circ} \mathrm{C}$. Entre diversos $\mathrm{GEI}$, el $\mathrm{CO}_{2}$ es el gas que más influye, considerándose como la causa inmediata del fenómeno del calentamiento global, por lo tanto, casi todos los estudios y esfuerzos para la disminución de la temperatura media mundial se está centrando en frenar las emisiones de $\mathrm{CO}_{2}$ de diversas fuentes emisoras; los sectores industriales son las principales fuentes de producción de $\mathrm{CO}_{2}$, en 2017, se liberaron 21.4 Gton $\mathrm{CO}_{2}$ de este sector que indica el impacto dramático de tales procesos sobre el calentamiento global [1] [2].

En respuesta a este impacto global, es necesario contar con sistemas robustos de administración de las emisiones de GEl para identificar las oportunidades de ahorro en el consumo energético y al mismo tiempo reducir las emisiones de GEI.

Actualmente, para las compañías y organizaciones se han desarrollado metodologías para realizar su inventario y reporte de emisiones GEI "Corporativo" este contexto se refiere a que la metodología es aplicable tanto para organizaciones públicas como privadas.

Las herramientas de cálculo son el complemento del estándar y ayudan a las empresas a cuantificar las emisiones provenientes de actividades. El protocolo de GEI [3], es la herramienta más utilizada a nivel internacional. Derivado de este estándar se han generado diversas herramientas de cálculo sectoriales, para la industria química, cemento, hierro, papel, entre otros.

El desafío del cálculo o inventario de huella de carbono, requiere una metodología sistemática y confiable para cumplir con los principios que establece el protocolo de GEI, con un mínimo de requisitos estandarizados que garanticen la obtención de resultados con alto nivel de confianza y un robusto soporte estadístico que asegure el nivel de calidad que permita la validación y verificación del informe de emisiones presentado [4].

Elementos para identificar y calcular emisiones [5]: Identificar fuentes, Selección del método de cálculo, Recolectar datos y seleccionar factores de emisión, Aplicar herramientas de cálculo, Enviar datos a nivel Corporativo.

Ante el complejo sistema para medir la huella de carbono [2] [6], se ha visto la necesidad de hacer la verificación y la auditoría externa, por lo que surgieron estándares en el mercado para la revisión de los inventarios, como: PAS2050 [2] [7], ISAE3410 [8], así como la serie ISO14064 parte 1-3[2][9], relativas a las cuantificaciones, reportes, la validación y verificación de Emisiones. En el presente proyecto se utilizará la parte 2 para la descripción del proyecto de control de emisión GEI como una primera base para la auditoria en una industria cementera.

Como consecuencia de la toma de conciencia social con respecto a los GEl y la necesidad de reducir estas emisiones, en los últimos años las empresas y otras entidades reclaman cada vez más estrategias y acciones que permitan compensar el impacto ambiental derivado del uso energético en los procesos de producciones de bienes y servicios.

Actualmente, en México empresas que verifican sus emisiones de GEI por auditoria tradicional de cumplimiento ambiental son escasas en el marco industrial. De acuerdo con el programa GEI México [10], 6 empresas en 2015 participaron en un esquema de certificación de desempeño de carbono, por lo que han realizado una verificación por una tercera parte independiente de su reporte de emisiones de GEl. 
La aprobación de la Ley General de Cambio Climático, ayuda a promover políticas e incentivos para reducir las emisiones de bióxido de carbono y el uso de combustibles de origen fósil y para impulsar las energías renovables.

¿Por qué la Industria de Cemento tiene el reto de administrar adecuadamente sus emisiones de GEI? Históricamente, el sector del cemento ha sido responsable de entre $5 \%$ y $9 \%$ de las emisiones globales de gases de efecto invernadero (GEI). En 2010, se emitieron más de 2800 millones de toneladas de GEI de la industria, una cifra que corresponde al $9 \%$ de las emisiones globales de $\mathrm{CO}_{2}$ [6][11]; con una producción anual de alrededor de 10 mil millones de toneladas en todo el mundo [12], siendo el cemento el producto de mayor consumo después del agua en todo el mundo, la producción de cemento es la más intensiva en energía y emisiones de la industria, sus características, físicas, las propiedades químicas y el proceso de fabricación han sido objeto de estudio durante mucho tiempo[6], actualmente se encuentra bajo presión para reducir las emisiones de GEI, sin embargo, reducir los GEl de la industria del cemento, no es una tarea fácil para países en vías de desarrollo [13].

El consumo per cápita se estima en 447 kg/hab y de acuerdo con la Federación Interamericana del Cemento (FICEM) [14], la producción mundial en 2009 fue de 3,033 millones de toneladas.

La industria cementera en un esquema global como resultado de las reacciones químicas en la producción de clinker, el consumo de combustible, el uso de electricidad y el transporte de materia primas y productos terminados representa un gran emisor de GEI [15] [6], esta industria proporciona soporte insustituible para el desarrollo sucesivo de otras industrias relacionadas, ya que el cemento es uno de los materiales de construcción más utilizados en el mundo, en ello radica también su importancia económica social [16].

En este contexto, tiene retos importantes la Industria Cementara en la administración de las emisiones de GEI. Actualmente a nivel global ha conformado una iniciativa global denominada: CSI (Cement Sustainable Initiative), que promueve al interior del sector cementero, la contabilidad y reporte de las emisiones de GEI, además de que estas emisiones sean auditadas por una tercera parte independiente [17].

México enfrentará retos importantes en el marco del cumplimiento de la Ley General de Cambio Climático, por tal razón, los programas de Auditoría Ambiental promovidos por la Procuraduría Federal de Protección Ambiental (PROFEPA), deberán crear sinergias para que los programas de auditoría ambiental consideren la administración de información no financiera como son las emisiones de GEl.

\section{Metodología}

Diseño del Proyecto de control GEI con base a ISO 14064-2 con visión a la auditoria en una Industria productora de cemento. Representa un elemento clave la auditoría de la huella de carbono para las empresas al considerar el marco de su desempeño ambiental. Garantizar un cálculo adecuado de las emisiones de GEl ayuda a las empresas a tener una mejor comprensión de la dimensión de sus emisiones, reconocer su potencial de ahorro de energéticos y establecer una base para la comparación y comunicación de los resultados alcanzados, tanto el interior como exterior [18].

En la práctica, la auditoria involucra la canalización del esfuerzo por parte del auditor hacia los datos y sistemas asociados que tengan en su calidad general. 
La auditoría de las emisiones GEI, involucra la evaluación de los riesgos de discrepancias materiales en los datos reportados. Las discrepancias ocurren entre los datos reportados y los datos generados por la aplicación apropiada de los estándares y metodologías de cálculo de las emisiones.

Cuando se realiza la auditoria para una Industria productora de cemento se emite una conclusión sobre si la información del Reporte de Emisiones de Gases de Efecto Invernadero se manifiesta de acuerdo al alcance, en particular para la industria del cemento es propuesto lo siguiente:

Alcance 1 (Emisiones Directas - Consumo de Combustible en obra y centro fijo)

Alcance 2 (Emisiones Indirectas- Asociadas a consumo de Energía Eléctrica en obra y centro fijo), y se encuentra libre de discrepancias en los cálculos, así como en los sistemas de reporte utilizados.

Por consiguiente, para el caso de la industria productora de cemento, es sumamente necesario homologar criterios de reporte para los GEl, contar con métodos claros y consistentes para evaluar, verificar y neutralizar las emisiones de sus productos a lo largo de su ciclo de vida.

\section{Método}

Como Metodología la norma ISO 14064 en la parte 2 en todos los aspectos de los proyectos de GEI proporciona una serie de cláusulas y facilita el seguimiento de los escenarios de línea base del proyecto contra el desempeño del proyecto y asegura que toda la información sea verificada y validada en el esquema de una auditoria ISO 14064-3.

En un resumen de la parte 2 de la ISO la metodología se sintetizaría en los elementos siguientes de la figura 1.
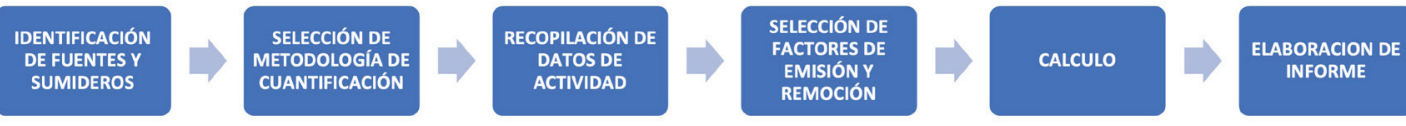

Figura 1. Resumen de la parte 2 de la ISO 14064-3. Fuente: [8].

Aquí presentamos la descripción del proyecto para una industria del cemento bajo la ISO 140642, esto es, describir el proyecto dando seguimiento a los requisitos de la norma, proporcionar elementos que sirvan como principio para determinar la línea base, los procedimientos de seguimiento listan algunos componentes para el diseño de los mismos, sugerir metodologías útiles para la industria del cemento en la cuantificación de emisiones, almacenamiento, reducciones de emisiones e incrementos de remociones $\mathrm{GEI}$, un prototipo para el contenido del informe y puntos de base para validar el proyecto de GEI, dado que la segunda fase queda fuera del objeto del presente trabajo, ya que es la etapa en la cual el proyecto debe ejecutar e implementar cada uno de los procedimientos, dar seguimiento y verificar el desempeño, Auditar para someterlo a la evaluación por terceros para buscar la certificación final.

\section{Objetivo general}

Proporcionar las bases en una Industria del sector cemento para la gestión de sus sistemas y procesos para el cálculo de los GEl alcance 1 y 2 y sugerir un procedimiento de cálculo para fortalecer el mecanismo de reporte de las emisiones GEl con la finalidad de Auditar las emisiones de Gases Efecto Invernadero (GEI). 


\section{Objetivos específicos}

- Identificar las fuentes de emisión de GEI de la Industria en su proceso de producción de cemento

- Identificar los procesos de reporte de emisiones de GEl alcance 1 y 2.

- Identificar los principales factores de la gestión del cálculo de las emisiones de GEI que tienen mayor incidencia en la contabilidad y reporte

Identificar los parámetros de calidad y credibilidad del reporte de la huella de carbono

\section{Identificación de variables}

El punto inicial es el establecimiento de la línea base que requiere de acordar al interior de la empresa fijar el año inicial o cero, e integrar un compendio histórico de al menos 5 años de las emisiones de GEl de la Industria que sean representativos de la actividad anual promedio, además todos los años deberán poder tratarse bajo el mismo esquema de análisis, cálculo y reporte, posteriormente se identificaran la variables con las que se habrá de trabajar. Para ello los mapas de proceso y una Check-list sería una herramienta de mucha utilidad.

\section{Diagrama Sagital}

Estos son diagramas usados para representar mediante flechas relaciones entre las partes de un conjunto los elementos de entrada y de partida. El marco del cálculo de las emisiones de GEI en el proceso de producción de cemento, involucran diversas variables entrantes y de partida a considerar, como muestra la figura 2.

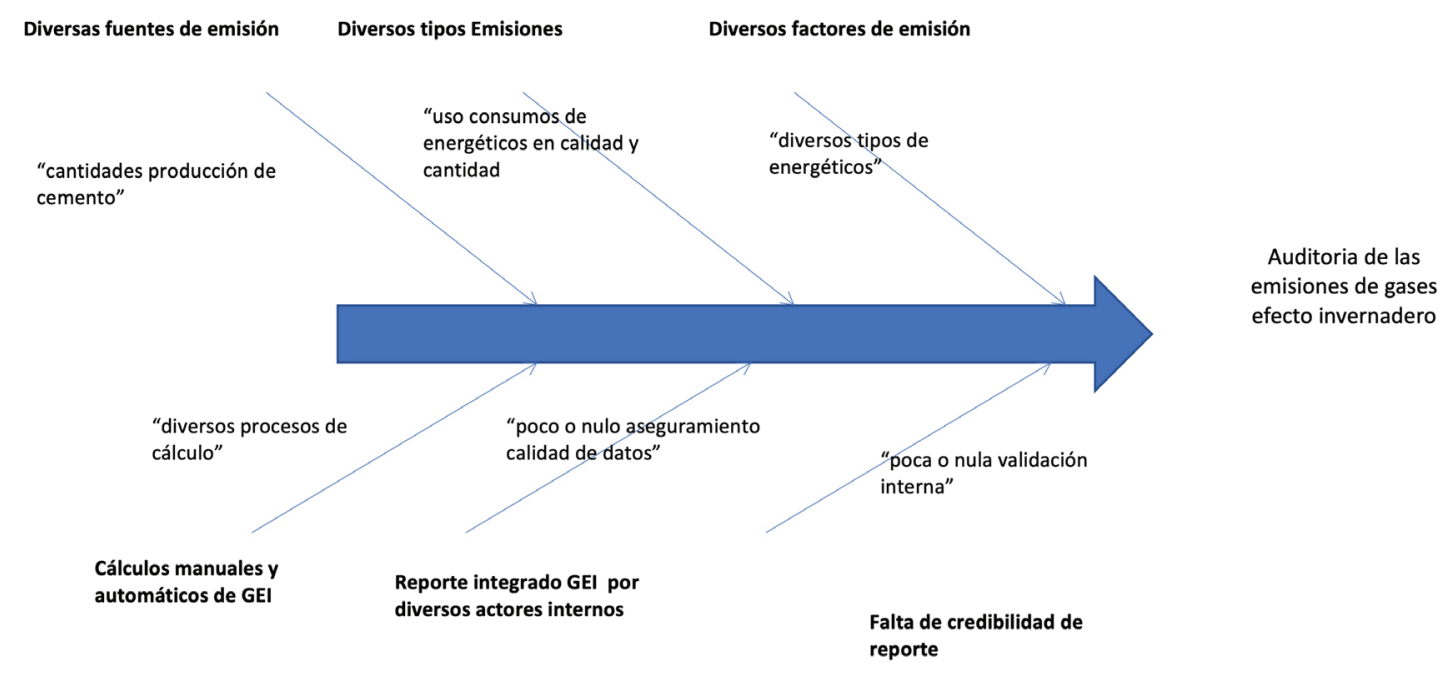

Figura 2. Diagrama Sagital del proceso de Auditoria de las emisiones de GEI..

La relación existente entre las variables permite seleccionar las esenciales para el proyecto, las variables consideradas permiten identificar los aspectos que son críticos en la administración de las emisiones de GEI, por lo que facilita el plantearse el "Problema" para la Auditoria de las emisiones GEl de una industria del Cemento en México. 
En el contexto del cálculo de las emisiones de GEI para la industria de producción de cemento, se describe como se propone realizar la identificación, los procesos de reporte de emisiones de GEl y de los principales factores de la gestión del cálculo de las emisiones de GEl, manifestando las diversas variables involucradas a considerar en el cuadro 1.

Cuadro 1. Gestión en el cálculo de las emisiones de GEl de la industria del cemento.

\begin{tabular}{|c|c|c|}
\hline Dominio & Indicadores & Definición teórica y operativa \\
\hline \multirow[t]{3}{*}{$\begin{array}{l}\text { Emisiones por } \\
\text { calcinación }\end{array}$} & $\begin{array}{l}\text { Producción de } \\
\text { Clinker }\end{array}$ & $\begin{array}{c}\text { El principal componente del cemento es el clínker. Este material } \\
\text { se produce a partir de materias primas naturales, como la caliza y } \\
\text { la arcilla, que se muelen, homogeneizan e introducen en un horno } \\
\text { rotatorio, donde se cuecen a una temperatura de } 1450^{\circ} \mathrm{C} \text {, necesaria } \\
\text { para formar el nuevo compuesto. } \\
\text { Los principales componentes del clínker son los óxidos de calcio. } \\
\text { Posteriormente, al clínker se le añade yeso y otros materiales } \\
\text { (escoria de alto horno, cenizas volantes, puzolanas, caliza, etc...) y } \\
\text { conjuntamente se trituran en un molino hasta formar un polvo fino: el } \\
\text { cemento, silicio, aluminio y hierro. }\end{array}$ \\
\hline & \multirow{2}{*}{$\begin{array}{l}\text { CKD y Bypass } \\
\text { Dusts que salen } \\
\text { del sistema del } \\
\text { horno }\end{array}$} & $\begin{array}{c}\mathrm{CO}_{2} \text { del polvo bypass o cement kiln dust (CKD) se liberan del sistema } \\
\text { del horno, deben ser calculados de acuerdo a volúmenes relevantes de } \\
\text { polvo y un factor de emisión. }\end{array}$ \\
\hline & & $\begin{array}{l}\text { Bypass dust es a menudo completamente calcinado. Por tanto las } \\
\text { emisiones relacionadas al bypass dust deben ser calculadas usando el } \\
\text { factor de emisión para el Clinker. }\end{array}$ \\
\hline \multirow{3}{*}{$\begin{array}{l}\text { Emisiones por } \\
\text { combustibles de } \\
\text { horno. }\end{array}$} & $\begin{array}{l}\text { Consumo de } \\
\text { combustible de } \\
\text { horno }\end{array}$ & Son los combustibles fósiles utilizados en el horno. \\
\hline & & $\begin{array}{l}\text { El poder calorífico es la cantidad de energía que la unidad de masa } \\
\text { de materia puede desprender al producirse una reacción química de } \\
\text { oxidación (quedan excluidas las reacciones nucleares, no químicas, de } \\
\text { fisión o fusión nuclear, ya que para ello se usa la fórmula } E=m c^{2} \text { ). }\end{array}$ \\
\hline & $\begin{array}{l}\text { Poder calorífico de } \\
\text { combustibles de } \\
\text { horno }\end{array}$ & $\begin{array}{c}\text { El poder calorífico expresa la energía máxima que puede liberar la unión } \\
\text { química entre un combustible y el comburente y es igual a la energía } \\
\text { que mantenía unidos los átomos en las moléculas de combustible } \\
\text { (energía de enlace), menos la energía utilizada en la formación de } \\
\text { nuevas moléculas en las materias (generalmente gases) formadas en } \\
\text { la combustión. La magnitud del poder calorífico puede variar según } \\
\text { como se mida. Según la forma de medir se utiliza la expresión poder } \\
\text { calorífico superior (abreviadamente, PCS) y poder calorífico inferior } \\
\text { (abreviadamente, PCl). }\end{array}$ \\
\hline \multirow{2}{*}{$\begin{array}{l}\text { Emisiones por } \\
\text { combustibles } \\
\text { fuera del horno }\end{array}$} & $\begin{array}{l}\text { Consumo de } \\
\text { combustible fuera } \\
\text { del hornos }\end{array}$ & $\begin{array}{l}\text { Es el combustible fósil que se usa fuera del horno tales como los que se } \\
\text { emplean en autos propios de la compañía, de la planta, del cuarto de } \\
\text { calentamiento, equipos para la preparación de componentes minerales. }\end{array}$ \\
\hline & $\begin{array}{l}\text { Poder calorífico de } \\
\text { combustibles fuera } \\
\text { del horno }\end{array}$ & Depende del tipo de hidrocarburo en consumo. \\
\hline \multirow[t]{2}{*}{$\begin{array}{l}\text { Producción de } \\
\text { productos de } \\
\text { cemento }\end{array}$} & $\begin{array}{c}\text { Componentes } \\
\text { minerales (MIC) } \\
\text { usados para } \\
\text { producir cementos } \\
\text { Portland y } \\
\text { mezclados }\end{array}$ & $\begin{array}{l}\text { MIC-componenetes minerales naturales o artificiales con propiedades } \\
\text { hidráulicas usadas como clinker o sustitutos de cemento }\end{array}$ \\
\hline & Clinker vendido & \\
\hline
\end{tabular}


Herramientas a utilizar

Información para alimentar el procedimiento de cálculo

- Mapas de proceso son una fuente de información relevante para detectar los puntos críticos

- Inventarios de emisiones calculadas por parte de la empresa

- Registro interno de CKD y polvos que salen del sistema del horno

- Facturas de clinker comprado y vendido, MIC comprado por parte de la empresa

- Niveles de stock registrados de Clinker y MIC

- Facturas de Combustibles

- Registro de la administración del stock interno y registro de la supervisión.

- Datos proporcionados por el laboratorio interno del análisis de calidad de los combustibles en la empresa

- Datos de Poderes caloríficos de combustibles proporcionado por el proveedor que abastece los combustibles.

- Datos de los análisis realizados por el Laboratorio externo

Check-list para la estructuración de los procedimientos de evaluación y auditoria.

Para el cálculo o evaluación en este mismo contexto, el protocolo de GEl ofrece diferentes herramientas para estimar la cuantificación de las emisiones de GEl en la producción de cemento, tales como: A cement-based tool requiring data only on annual cement production. Emissions are calculated using clinker: cement ratios así como la herramienta de CSI (Cement Sustainability Initiative): A clinker based tool suitable for when the amount of clinker consumed.

De acuerdo con el Protocolo de GEl, en la definición de la calidad de los inventarios de emisiones, se destacan cinco principios de confiablidad:

1. 1. Relevancia, que está relacionado con los límites del inventario;

2. 2. Integridad, todas las emisiones dentro de un límite de inventario definido requiere ser contabilizadas;

3. 3. Consistencia, la aplicación permanente de la contabilidad, el límite de los inventarios y las metodologías esenciales.

4. 4. Transparencia, se refiere al grado en qué la información relacionada a los procesos, procedimientos, suposiciones y limitaciones de los inventarios de GEl es presentada de manera clara, efectiva, neutral y comprensible, basada en documentación sólida, transparenté y auditable; con precisión, para que los datos deban ser suficientemente precisos como para tomar decisiones.

5. 5. La medición de GEl y las estimaciones o cálculos nunca deben presentar errores sistemáticos sobre o por debajo del valor real de las emisiones.

Etapa final la auditoría de la huella de carbono se realiza con base a los criterios de la ISO14064-3[8], la cual establece los requisitos para la verificación de los inventarios y proyectos relacionados a los GEI. Además, describe el proceso para la verificación de las emisiones de GEI, y específica los mecanismos de planificación y procedimientos de evaluación, y las declaraciones que se realizan ya sea por la organización o del proyecto de GEl. El resumen de la propuesta metodológica de revisión por la auditoria basada en ISO 14064-3 consistiría en figura 3. 


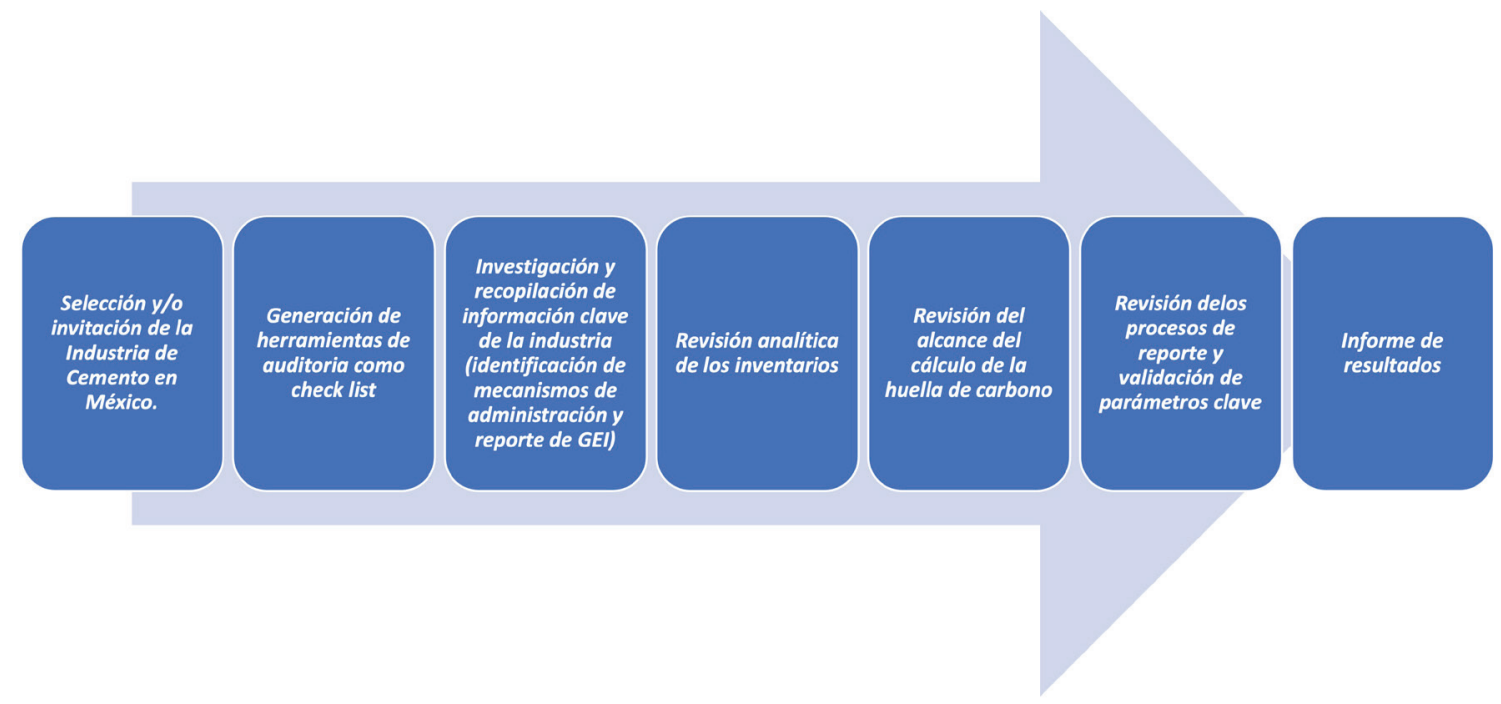

Figura 3. Propuesta metodológica de revisión por la auditoria basada en ISO 14064-3.

En la auditoria se evaluará el riesgo de discrepancia material de cada uno de los componentes, los procesos de recopilación y reporte de información GEl. Esta evaluación es utilizada para planificar y dirigir el proceso de verificación. Para evaluar este riesgo, se considerará los factores siguientes:

- La estructura de la organización y el enfoque de la organización utilizado para asignar la responsabilidad de monitorear y reportar las emisiones.

- El método de aproximación y el compromiso de la administración con el monitoreo reporte de GE.

- La complejidad de los sistemas para procesar la información.

- El estado de calibración y mantenimiento de medidores utilizados.

- La confiabilidad y disponibilidad de los datos ingresados.

- El desarrollo e implementación de políticas y procesos para el monitoreo y reporte.

- Las suposiciones y estimaciones aplicadas.

\section{Resultados}

La gestión de los resultados tiene fundamento en uno de los objetivos de mayor importancia del proyecto que es identificar los parámetros de calidad y credibilidad del reporte de la huella de carbono.

Además, a la hora de reportar las emisiones directas de los GEl es necesario hacerlo, con un alto grado de detalle para cada caso de GEl. Por otro lado, las emisiones de $\mathrm{CO}_{2}$ por combustión de biomasa siempre se considera que deberán ser contabilizadas aparte, el reporte de las emisiones, se realiza en toneladas de CO2-e, diferenciando cada categoría.

Como lograr integrar una gran cantidad de información sin que se escapen los detalles sugerimos una forma que puede ser estructurar una matriz con la información colectada por área, colocando el proceso y cada una de sus etapas Vs el consumo en movilidad y fuente fija con ello las emisiones correspondientes, lo que posteriormente facilita la elaboración de las hojas de cálculo, cuadro 2. 
Cuadro 2. Propuesta de matriz de resultados.

\begin{tabular}{|c|c|c|c|c|c|c|c|}
\hline $\begin{array}{c}\text { Emisiones } \\
\text { directas }\end{array}$ & \multicolumn{2}{|c|}{ Consumo de gas } & \multicolumn{2}{|c|}{$\begin{array}{c}\text { Consumo de } \\
\text { combustible } \\
\text { (TJ) }\end{array}$} & $\begin{array}{c}\text { Consumo Energía } \\
\text { eléctrica } \\
\text { (kWh) }\end{array}$ & $\begin{array}{c}\text { Emisiones } \\
\text { totales GEl }\end{array}$ \\
\hline Proceso & Obra móvil & Centro fijo & Obra móvil & $\begin{array}{c}\text { Centro } \\
\text { fijo }\end{array}$ & Obra móvil & $\begin{array}{c}\text { Centro } \\
\text { fijo }\end{array}$ & \\
\hline & & & & & & \\
\hline
\end{tabular}

En la generación de datos de calidad cuando se tiene una evaluación cuantitativa se precisa del cálculo de incertidumbre siempre que sea técnica y económicamente viable, no obstante en el caso de las emisiones GEl esto no siempre será así. Por lo que de acuerdo con Unzalu [18], en la guía metodológica para la aplicación de la norma ISO-14064 propone que la organización puede establecer un enfoque cualitativo para analizar el impacto de la incertidumbre de los datos presentados en el informe de GEl y recomienda solo realizar el cálculo matemático de incertidumbre cuando:

- Existe un requisito legal por ejemplo aquellas organizaciones a las que les afecte el esquema europeo de comercio de emisiones (eUeTS)

- El caso en cual el seguimiento de los datos de actividad con mayor peso en el cómputo total de GEI se realiza con contadores internos de la organización.

En la gestión de los resultados contar con un sistema de registro en el cual se capturen toda la información y cada uno de los datos usados en las memorias de cálculo es de suma importancia como apoyo para el sistema de registro en el anexo 1 se ha diseñado el prototipo para realizar la auditoria en el cual se podrá evidenciar el desempeño del proyecto de control para GEI y se facilitan pasos, como:

- Identificar de las brechas en el marco del cálculo de la huella de carbono y ejecución de un plan de acción para el cierre de brechas

- La Identificación del nivel de madurez de los procesos de la empresa con relación a la administración de la huella de carbono.

- Procedimiento (protocolo) de reporte de indicadores GEl para la homologación de los criterios de cálculo y reporte

Reporte de Indicadores de GEI

\section{Conclusiones}

Lo que aquí presentamos es una guía que toma la industria cementera como ejemplo para realizar una metodología para el cálculo de emisiones de gases efecto invernadero y de cómo puede inventariar esas emisiones, revisar sus procesos para encontrar las áreas de oportunidad, que desde un enfoque empresarial esto representan un ahorro económico donde a la larga la inversión inicial puede ayudar a disminuir sus costos de producción y contribuir a mitigar los daños al medio ambiente.

El cambio climático representa un reto para las industrias en general y no solo en México, en todo el mundo, las políticas públicas ambientales así como la legislación diseñada para atender este fenómeno resulta un diseño útil para que las empresas comiencen a cambiar sus procesos, 
ya sea por reacción y cumplimiento al marco regulatorio o por el convencimiento cultural, al invertir en nuevas tecnologías, en auditorias, en cálculos de inventarios, ayudan al medio ambiente, a su entorno, y las transforma en empresas sustentables, y no solo forman parte de la larga fila de empresas maquilladas con verde.

Con este último objetivo de aportar criterios de valoración, que adicionalmente debieran ser estandarizados fue desarrollado el presente trabajo como un modelo, diseñado para la industria cementera, por ser una de las más contaminantes y mayor emisora de GEI, pero esta metodología podrá ser implementada en otro tipo de industrias, no obstante la implementación de la normatividad diseñada de manera internacional, requiere de adecuarse en México, de la contratación de expertos o bien el conocimiento mínimo de las normas, su interpretación y su implementación, así como el desarrollo de indicadores, el análisis de los procesos y la elección precisa de las variables será producto solo del entrenamiento y la mejora continua en cada industria.

Se considera que una de las principales dificultades para el diseño de los proyectos GEl se encuentra en la recopilación, la gestión de la información y control de todos aquellos datos, como los derivados del transporte de materia prima o salida de producto, dado que estos no los gestiona directamente el proyecto (Industria del cemento $\mathrm{GEI}$ ) pero son fundamentales porque establecen los límites del sistema de cuantificación la frontera de dónde empezar y terminar el proyecto.

\section{Agradecimientos}

Trabajo derivado del proyecto de investigación SIP 20195759, patrocinado por el Instituto Politécnico Nacional.

\section{Referencias}

[1] E. Benhelal, E. Shamsaei, M.I. Rashid. "Challenges against CO2 abatement strategies in cement industry: A review", Journal of Environmental Sciences, Volume 104, 2021, Pages 84-101, https://doi.org/10.1016/j. jes.2020.11.020

[2] N.C.Onat, M. Kucukvar. "Carbon footprint of construction industry: A global review and supply chain analysis", Renewable and Sustainable Energy Reviews, Volume 124, 2020, 109783, https://doi.org/10.1016/j. rser.2020.109783

[3] P. Wu, B. Xia, J. Pienaar, X. Zhao. "The past, present and future of carbon labelling for construction materials - A review" Building and Environment, Volume 77, 2014, Pages 160-168, https://doi.org/10.1016/i.buildenv.2014.03.023

[4] Gases de Efecto Invernadero (GEI). Available: https://ghgprotocol.org/

[5] Green house gas protocol. Available: https://ghgprotocol.org/guidance-0

[6] J. Cagiao, B. Gómez, J.L. Doménech, S. Gutiérrez Mainar, H.Gutiérrez Lanza. "Calculation of the corporate carbon footprint of the cement industry by the application of MC3 methodology". Ecological Indicators, Volume 11, Issue 6, 2011, Pages 1526-1540, https://doi.org/10.1016/i.ecolind.2011.02.013

[7] British Standards Institution, BSI . Guide to PAS 2050. How to assess the carbon footprint of goods and services. 2008

[8] International Atandard on Assurance Engagements 3410 (ISAE 3410), Assurance engagements on greenhouse gas statements. Available: http://www.ifac.org

[9] International Organization for Standarization. Greenhouse Gases -Part 3: specification with guidance for the validation of greenhouse gas assertions, (ISO 14064), firts edition 2006-03-01.

[10] GEl México. Available: http://www.geimexico, consultado el 9 de octubre d 2012.

[11] A. Talaei, D. Pier, A. V. Iyer, Md Ahiduzzaman, A. Kumar. "Assessment of long-term energy efficiency improvement and greenhouse gas emissions mitigation options for the cement industry", Energy, Volume 170, 2019, Pages 1051-1066, https://doi.org/10.1016/j.energy.2018.12.088. 
[12] E. Hache, M. Simoën, G. Sokhna Seck, C. Bonnet, A. Jabberi, S. Carcanague." The impact of future power generation on cement demand: An international and regional assessment based on climate scenarios". International Economics, Volume 163, 2020, Pages 114-133, https://doi.org/10.1016/i.inteco.2020.05.002.

[13] S. Balsaraa, P. Kumar Jainc, A. Rameshd. "An integrated approach using AHP and DEMATEL for evaluatingclimate change mitigation strategies of the Indian cement manufacturing industry", Environmental Pollution 252 (2019) 863-878, https://doi.org/10.1016/i.envpol.2019.05.059

[14] Federación Interamericana del Cemento (FICEM) Informe estadístico 2011, FICEM.

[15] CEMEX (2010), Inventario Corporativo de GEI Cemex México.

[16] D. Song, B. Chen." A Life Cycle Modeling Framework for Greenhouse Gas Emissions of Cement Industry", Energy Procedia, Volume 61, 2014, Pages 2649-2653,

https://doi.org/10.1016/j.egypro.2014.12.267

[17] J. Farfan, M. Fasihi, C. Breyer. "Trends in the global cement industry and opportunities for long-term sustainable CCU potential for Power-to-X”, Journal of Cleaner Production, Volume 217, 2019, Pages 821-835,

https://doi.org/10.1016/i.jclepro.2019.01.226

[18] M. Schneider."The cement industry on the way to a low-carbon future", Cement and Concrete Research, Volume 124, 2019, 105792,

https://doi.org/10.1016/i.cemconres.2019.105792

[19] Unzalu, P. Guía Metodológica para la aplicación de la norma UNE ISSO 14064-1:2006 para el desarrollo de inventarios de gases de efecto invernadero en organizaciones. Ed. Ihobe, Sociedad Pública de Gestión Ambiental. España. 2012 


\section{Anexos}

\section{Anexo 1. Prototipo para un sistema de registro para el control GEI}

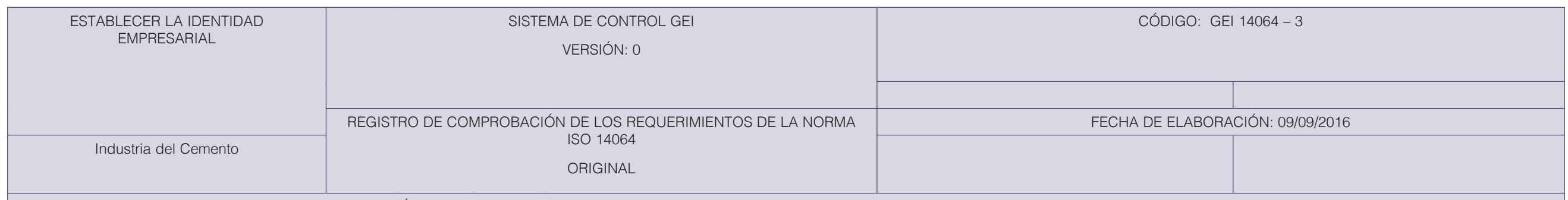

PROPÓSITO: Comprobar el cumplimiento de los requisitos de la ISO 14064:2 para verificar y validar la información vía la auditoria

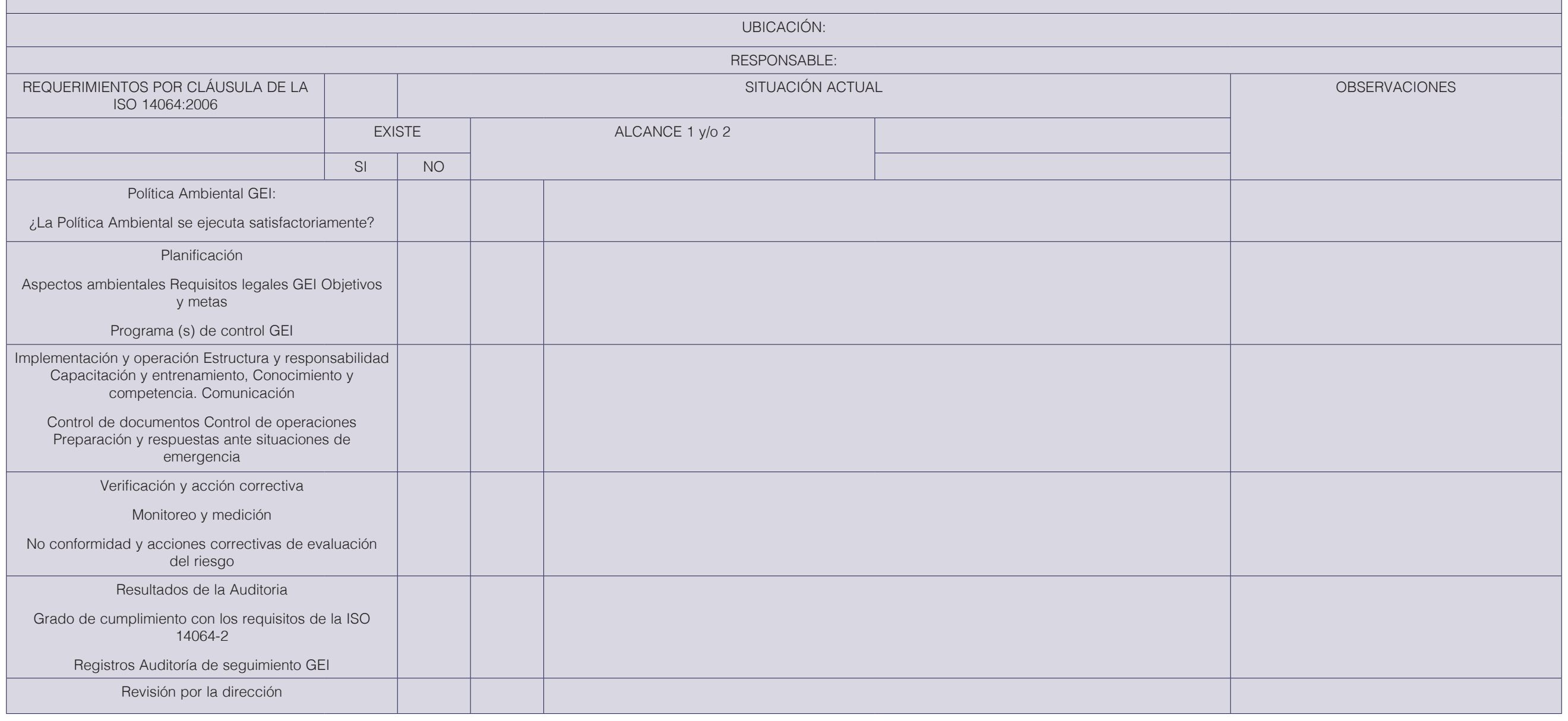

